\title{
Image Shifts Caused by Rotating a Constant- Deviation Prism in Divergent Light
}

\author{
By James B. Saunders
}

\begin{abstract}
The use of the constant-deviation prism for producing monochromatic light introduces the problem of image shifts that result from rotating the prism when changing from one spectral line to another. The magnitudes of both the lateral and the axial components of this shift, for a given rotation of the prism, depend upon the position of the axis about which the prism rotates. A position for the axis is known that yields zero lateral shift. The axial shift, however, depends also upon the convergence of the beam and the shape and optical properties of the prism. In this paper a method is presented for locating the positions of the axis of rotation for minimum axial and for minimum total shifts of the foci for both the sagittal and meridian fans of rays.
\end{abstract}

\section{Introduction}

The constant-deviation prism, as used in a parallel beam of light emerging from the collimator of a spectroscope or monochromator, has been discussed by Uhler [1, 2], ${ }^{1}$ Forsythe [3], and Block [4]. These authors were interested only in the lateral movement of the beam that occurs when the prism is rotated, as in changing from one spectral line to another. They gave no consideration to the case of convergent or divergent light and, therefore, ignored the possibility of axial or lateral changes in the position of the image. Such displacements did not enter their problem, because there is no change in the position of the image if the rays of light within the prism remain parallel. As a result of their studies for the case of collimated light, a unique position of the axis of rotation for which no lateral shift of the beam occurs is known. In this paper it is shown that the same axis serves for noncollimated light as well.

The magnitude of the lateral shift of a converging or diverging beam of light is a measure of the lateral shift of the image. Consequently, the lateral position of the beam and the axial positions of the primary and secondary images, as functions

\footnotetext{
1 Figures in brackets indicate the literature references at the end of this pa-
} per.

of the angular position of the prism, determine the movements of the image point when the prism is rotated. A simultaneous treatment of these three functions shows that any two of the shifts may be practically eliminated by a proper selection of the axis of rotation.

In most applications of the constant deviation prism a slit-like source (or its equivalent) is used, and one of the astigmatic images either becomes of secondary importance, or its axial movements may be ignored. In this paper a particular application of the constant deviation prism is considered in which one or the other of these images may be ignored. The treatment applies to the recently designed interferograph [5], an instrument for photographing the movements of interference fringes. A constant deviation prism and a single achromatic lens are used to form a monochromatic image of the interference pattern on a photographic film at a fixed distance. The derived equations apply to any triangular constant deviation prism, and particular properties are assigned for an illustration of results.

\section{Statement of the Problem}

An investigation of the axial image shifts caused by rotating the prism is facilitated by treating the 
meridian (Southall's terminology ${ }^{2}$ used) and sagittal rays separately. The primary and secondary images of a plane that are produced by these two beams usually do not coincide, nor is the shift of the two images equal for a given rotation of the prism about a specified axis. The shape and orientation of the source that illuminates the object plane may, and usually do, contribute differently to the astigmatic effect. Assuming the object to be a point on an interferometer plate that is illuminated by collimated light from a slit-like source in the focal plane of the collimator, a cross section of the beam reflected from this point will approximate a line. Whenever such a beam passes through a prism and a single lens to produce a real image of the point, the astigmatic effect caused by the prism depends upon its orientation with respect to the slit-like source. If the slit-like source is parallel to the refracting edge of the prism, the sagittal rays are more divergent than the meridian rays. The difference in divergence is reversed if the refracting edge is perpendicular to the source. In either case, the less divergent beam produces an image with a comparatively large depth of focus, whereas the depth of focus for the more divergent beam is relatively small. Consequently, the beam that has the greater divergence requires more consideration.

A detailed treatment of this problem of the lateral and axial shifts is too extensive to be given here. However, a presentation of the fundamental equations and their application to a typical, but particular, case furnished the necessary basis for their application to other particular cases. The equations of condition form a system of simultaneous equations, the solution of which gives all the information necessary for a solution in any special application. Either of two slightly different methods of attacking the problem may be followed. One is to require the differential of the shifts with respect to the angle of rotation of the prism to be zero at some specified wavelength of light. Usually this wavelength should be chosen near the middle of the visible spectrum. The second method requires the equality of the image distances for two wavelengths, one near each end of the visible spectrum. The second of these

2 The "meridian rays" are those refracted rays of an infinitely narrow homocentric bundle of incident rays that lie in a principal plane of the prism. The "sagittal rays" are those that lie in a plane containing the chief refracted ray and perpendicular to the principal plane. procedures was chosen because it seemed somewhat simpler for this particular presentation.

The following is a sufficient statement of the general problem for its analytical treatment. A source ${ }^{3}$ of light is located on the axis and in the left focal plane of the achromatic lens shown in figure 1. The point at which the axial ray emerges from the lens is chosen as the origin of a plane oblique coordinate system with the $x$-axis coinciding with the axis of the lens. A constant deviation prism is placed with the center of its entrance face initially at the point $(G, O)$ and with its refracting edge normal to the plane of the coordinate axes. Collimated light, from the lens, falls on the first or entrance face of the prism, is internally reflected at the second face, and emerges from the third or exit face at various angles, depending upon the angle of incidence and the geometrical and optical properties of the prism. This discussion is limited to those rays whose angle of emergence, $\theta$, equals the angle of incidence, a condition corresponding to minimum deviation. The total deviation of the rays that emerge under this condition is constant regardless of their wavelengths, and is equal to the angle of the prism as shown in figure 1 .

For the initial conditions of the analytical treatment, it is assumed that the angular position, $\theta_{2}$, of the prism corresponds to the known refractive index for wavelength, $\lambda_{2}$, near the middle of the visible spectrum. The indices for two other wavelengths, $\lambda_{1}$ and $\lambda_{3}$, one near each end of the visible spectrum, must also be known. After the prism has been placed in the above initial position, $\theta_{2}$, all further movements are limited to rotation about some specified axis that is normal to the plane of coordinates. The position of the prism shown in figure 1 is the result of an arbitrary rotation from the initial position. The angle between the positive $x$ - and $y$-axes is chosen equal to the internal angle, $\omega$, of the prism that lies opposite the reflecting face so that the emergent ray will be parallel to the $y$-axis.

The principal emergent ray is intercepted normally by a stationary interferometer plate. This point of interception and reflection $\left(X_{5}, Y_{5}\right)$ is considered the object point. The principal ray returns from the interferometer along its previous path and continues along the axis of the lens to the conjugate plane of the interferometer. The

\footnotetext{
${ }^{3}$ Such a source can be virtual and formed by a plane, half-silvered mirror that is so orientated that it reflects the light from a real source to the lens.
} 
image point, therefore, remains on the axis of the lens regardless of changes caused in the position of the object point by rotating the prism. Any lateral change in the position of the object point is relative to the fixed interferometer and therefore corresponds to a lateral change in the position of the image of the interferometer. Also, a change in the effective object distance caused by rotating the prism produces a corresponding change in the axial or longitudinal position of the image distance. Consequently, the lateral shifts and effective object distances of the object point are a measure of the lateral and longitudinal shifts, respectively, of the image of the interferometer. The correspondence between image and object movements are so well known that this discussion will be limited to movements of the object point alone. The problem then is to locate the position, $(X, Y)$, of the axis of rotation that will result in a minimum shift of the object point as the wavelength of the light, falling normally on the interferometer, changes from $\lambda_{1}$ to $\lambda_{3}$.

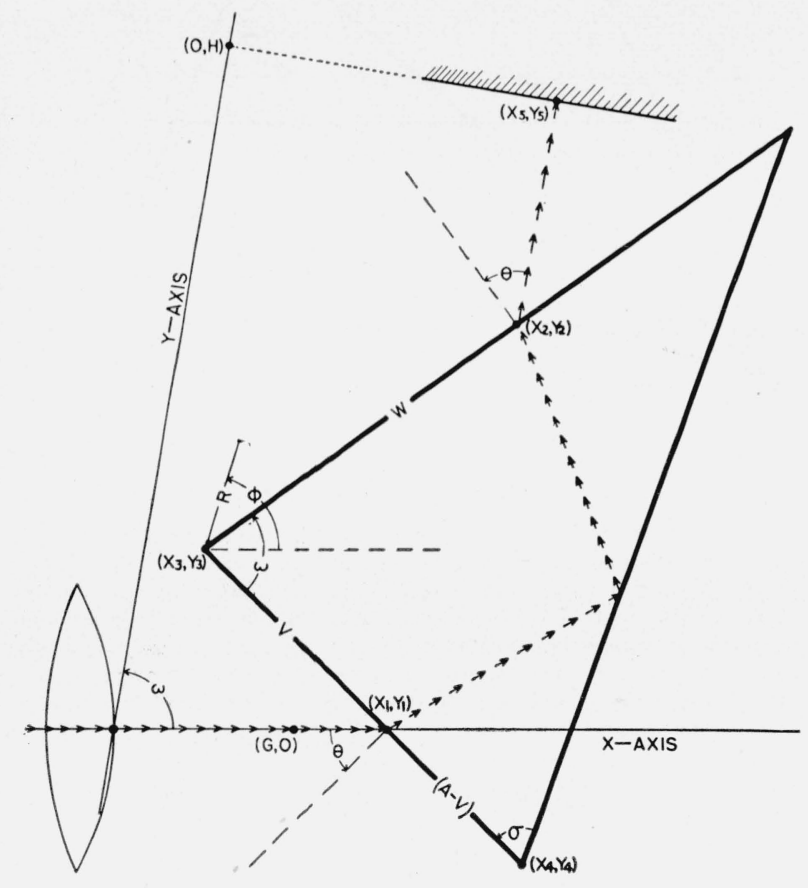

Figure 1. Diagram showing the relative position of optical parts.

The collimating lens, constant deviation prism, and the interferometer plate are schematically represented. The coordinates of the points that are indicated by dots are represented by the symbols in the adjacent parenthesis. The sum of the segments $V$ and $(A-V)$ is $A$, the length of the entrance face of the prism, and is the unit of length on which all the computations are based.

\section{Symbols and Abbreviations}

The solution of this problem requires a considerable number of symbols. The definition of those that have not already been given or that are not obvious from the figures are listed below.

$\lambda_{1}, \lambda_{2}$, and $\lambda_{3}$ are wavelengths of light corresponding to known indices of refraction of the prism. Usually these correspond to the $C, D$, and $F$ Fraunhofer lines. $\theta_{1}, \theta_{2}$, and $\theta_{3}$ are angles of incidence (or angles of emergence) corresponding to wavelengths $\lambda_{1}, \lambda_{2}$, and $\lambda_{3}$, respectively. $\theta_{m}$ is the value of $\theta$ when $\delta X_{5} / \delta \theta=0 . \quad A$ is the length of entrance face of prism. $L$ is the path length of principal ray in prism.

\section{$L_{2}=$ value of $L$ for $\lambda=\lambda_{2}$}

$P=$ effective object distance for the sagittal rays

$P \nu=$ value of $P$ for $\lambda=\lambda_{\nu}(\nu=1,2$, or 3$)$

$\Delta P=$ absolute magnitude of difference between largest and smallest values of $P$ in the range from $\theta_{1}$ to $\theta_{3}$

$Q=$ effective object distance for the meridian rays

$R=$ distance from vertex of angle $\omega$ to the axis of rotation

$V=$ distance from $\left(X_{3}, Y_{3}\right)$ to entrance point of axial or principal ray

$V_{2}=1 / 2 A$ and is the value of $V$ for $\lambda=\lambda_{2}$

$W=$ distance from $\left(X_{3}, Y_{3}\right)$ to exit point of principal ray

$W_{2}=$ value of $W$ for $\lambda=\lambda_{2}$, defined in eq $14^{\prime}$

$\left(X_{c}, Y_{c}\right)=$ coordinates of the "critical point," defined in the text

$\left(X_{i}, Y_{i}\right)=$ coordinates of the "intersection point," defined in the text

$X_{5 \nu}=$ value of $X_{5}$ for $\lambda=\lambda_{\nu}(\nu=1,2$, or 3$)$

$\Delta X_{5}=$ absolute magnitude of difference between largest and smallest values of $X_{5}$ in the range from $\theta_{1}$ to $\theta_{3}$.

\section{Derivations}

The following relationships are obtained from figure 1:

$$
\begin{gathered}
X_{1}-X_{3}=V \cos (\theta-\omega) \csc \omega, \\
Y_{3}-Y_{1}=V \cos \theta \csc \omega, \\
X_{2}-X_{3}=W \cos \theta \csc \omega
\end{gathered}
$$




$$
\begin{gathered}
Y_{3}-Y_{2}=W \cos (\theta+\omega) \csc \omega, \\
X-X_{3}=R \sin (\omega-\phi) \csc \omega, \\
Y-Y_{3}=R \sin \phi \csc \omega, \\
X_{2}=X_{5}, \\
Y_{5}=H-X_{5} \cos \omega,
\end{gathered}
$$

The principal ray coincides with the $x$-axis, therefore,

$$
Y_{1}=0 \text {, }
$$

and since the prism must rotate as a rigid body, about the axis of rotation,

$$
\theta-\theta_{2}=\phi-\phi_{2},
$$

$\left(\theta_{2}\right.$ and $\phi_{2}$ being the values for $\theta$ and $\phi$ for the initial conditions mentioned above).

The object distance for the sagittal rays is the sum of the equivalent air path of the principal ray [6] in glass, and the path in air, or

$$
P=X_{1}+Y_{5}-Y_{2}-L \cos (\sigma+1 / 2 \omega) \csc \theta
$$

The corresponding distance for the meridian rays is [6]

$$
\begin{array}{r}
Q=X_{1}+Y_{5}-Y_{2}-L \cos ^{2} \theta \csc \theta \\
\cos (\sigma+1 / 2 \omega) \csc ^{2}(\sigma+1 / 2 \omega)
\end{array}
$$

The values for $L$ and $(V+W)$, both of which are derived from figure 2 by applying the law of sines to the several triangles formed in the figure, are found to be

$$
\begin{aligned}
& L=2 V \cos \left(\sigma+\frac{1}{2} \omega\right)- \\
& 2 A \sin (\sigma) \cos (\sigma+\omega) \csc \left(\sigma+\frac{1}{2} \omega\right),
\end{aligned}
$$

and

$$
V+W=2 A \sin \sigma \cos \frac{1}{2} \omega \csc \left(\sigma+\frac{1}{2} \omega\right) \ldots
$$

The application of Shell's law to this particular problem gives the equation

$$
\sin \theta=-n \cos \left(\sigma+\frac{1}{2} \omega\right) \ldots
$$

from which the values of $\theta_{1}, \theta_{2}$, and $\theta_{3}$, corresponding to $\lambda_{1}, \lambda_{2}$, and $\lambda_{3}$, respectively, are obtained.

The above equations, 1 to 15 , are sufficient to derive the abscissa, $X_{5}$, of the object point; the object distance, $P$, for the sagittal rays; and the object distance, $Q$, for the meridian rays, in terms of known constants and the parameters $\theta, X$, and $Y$.

The interferograph uses a slit-like source of light that is parallel to the refracting edge of the prism; consequently, the effect of the meridian rays is negligible in comparison to that of the sagittal rays. The equation for evaluating $Q$ is therefore dropped from further consideration in this discussion. Should it be desirable to study $Q$, its treatment would be quite similar to that which will be followed here for $P$.

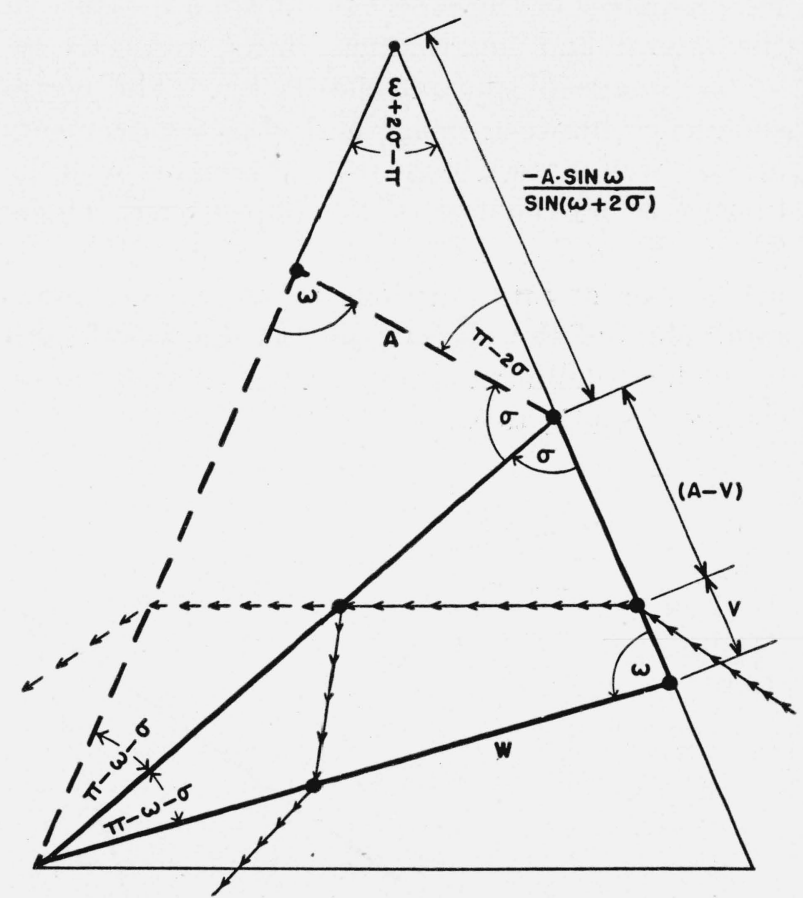

Figure 2. Geometrical correspondence between equivalent isosceles and constant deviation prisms.

The constant deviation prism is represented by the heavy line enclosure, with its reflecting face opposite the internal angle $\omega$. The mirror image of this prism, as reflected in its reflecting face, is completed with the heavy broken lines. The image of the exit face coincides with the exit face of the corresponding isosceles prism, which is completed with light lines. Consequently, the path in glass for any given ray of light is the same for either prism and depends upon the dimensions and shape of the prism, the position of the entrance point, and the angle of incidence.

\section{Illustrative Application}

To illustrate the solution of this problem for an important particular case, a constant deviation prism of the Pellin and Broca [7] type is selected that has refractive indices such that $\theta_{1}, \theta_{2}$, and $\theta_{3}$ are $54^{\circ}, 55^{\circ}$, and $56^{\circ}$, respectively. The values of $\omega$ and $\sigma$ are $90^{\circ}$ and $75^{\circ}$, respectively. By substituting the angles of this prism into the general equations and eliminating $Y_{1}$ and $X_{2}$ by means of 
eq 7 and 9 , they reduce to the corresponding prime numbered equations given below.

$$
\begin{gathered}
X_{3}=X_{1}-V \sin \theta, \\
Y_{3}=V \cos \theta, \\
X_{2}=X_{5}=X_{3}+W \cos \theta, \\
Y_{2}=Y_{3}+W \sin \theta, \\
R \cos \phi=X-X_{3}, \\
R \sin \phi=Y-Y_{3}, \\
Y_{5}=H, \\
\phi=\theta+\phi_{2}-\theta_{2}, \\
P=X_{1}+Y_{5}-Y_{2}+\frac{1}{2} L \csc \theta, \\
L+V=A\left(1+\frac{2}{\sqrt{3}}\right)=L_{2}+\frac{1}{2} A, \\
W+V=A\left(1+\frac{1}{\sqrt{3}}\right)=W_{2}+\frac{1}{2} A, \\
n=2 \sin \theta .
\end{gathered}
$$

On eliminating $X_{3}, Y_{3}$, and $W$ by substitution from eq $1^{\prime}, 2^{\prime}, 3^{\prime}, 4^{\prime}$, and $14^{\prime}$, the following equations were obtained:

$$
\begin{gathered}
X_{5}=X_{1}-V \sin \theta+\left(W_{2}+\frac{1}{2} A-V\right) \cos \theta, \\
Y_{2}=V(\cos \theta-\sin \theta)+\left(W_{2}+\frac{1}{2} A\right) \sin \theta .
\end{gathered}
$$

Similarly, by eliminating $X_{3}, Y_{3}$, and $\phi$ from eq $1^{\prime}$, $2^{\prime}, 5^{\prime}, 6^{\prime}$, and $10^{\prime}$, it follows that

$$
\begin{gathered}
X_{1}=V \sin \theta+X-R \cos \left(\theta+\phi_{2}-\theta_{2}\right), \\
V \cos \theta=Y-R \sin \left(\theta+\phi_{2}-\theta_{2}\right) .
\end{gathered}
$$

On substituting the value of $V$ from eq 19 into eq $13^{\prime}$ and 18 , they become

$$
\begin{gathered}
L=L_{2}+\frac{1}{2} A+\left[R \sin \left(\theta+\phi_{2}-\theta_{2}\right)-Y\right] \sec \theta, \\
X_{1}=X+Y \tan \theta-R \cos \left(\phi_{2}-\theta_{2}\right) \sec \theta
\end{gathered}
$$

The value of $V$ in eq 19 and $X_{1}$ in eq 21 are substituted into 16 and 17 to give the following:

$$
\begin{aligned}
& X_{5}=X-Y+R\left[\sin \left(\theta+\phi_{2}-\theta_{2}\right)-\right. \\
& \left.\cos \left(\theta+\phi_{2}-\theta_{2}\right)\right]+\left(W_{2}+\frac{1}{2} A\right) \cos \theta,
\end{aligned}
$$

$$
\begin{aligned}
& Y_{2}=(1-\tan \theta)\left[Y-R \sin \left(\theta+\phi_{2}-\theta_{2}\right)\right]+ \\
& \left(W_{2}+\frac{1}{2} A\right) \sin \theta .
\end{aligned}
$$

By replacing $Y_{5}, L, X_{1}$, and $Y_{2}$ in eq $11^{\prime}$ by their equivalents in eq $8^{\prime}, 20,21$, and 23 , respectively, the equation for $P$ becomes

$$
\begin{aligned}
& P=X+Y(2 \tan \theta-1-\csc 2 \theta)+\mathrm{H}+ \\
& \left(W_{2}+\frac{1}{2} A\right) \sin \theta+\frac{1}{2}\left(L_{2}+\frac{1}{2} A\right) \csc \theta+ \\
& R\left[(1-2 \tan \theta+\csc 2 \theta) \sin \left(\theta+\phi_{2}-\theta_{2}\right)-\right. \\
& \left.\cos \left(\theta+\phi_{2}-\theta_{2}\right)\right] .
\end{aligned}
$$

According to the above-described initial position of the prism for wavelength $\lambda_{2}$, it follows that for this position $\theta=\theta_{2}, X_{1}=G$, and $V=\frac{1}{2} A$. By applying these initial conditions to eq $1^{\prime}, 2^{\prime}$, $5^{\prime}$, and $6^{\prime}$, and by eliminating $X_{3}$ and $Y_{3}$, solutions are obtained for the initial values of $R$ and $\phi$ (i. e., $R_{2}$ and $\phi_{2}$ ) in terms of known constants and the coordinates, $(X, Y)$, of the axis of rotation. They are

$$
\begin{gathered}
\tan \phi_{2}=\frac{2 Y-A \cos \theta_{2}}{2 X-2 G+A \sin \theta_{2}} \\
R^{2}=\left(X-G+\frac{1}{2} A \sin \theta_{2}\right)^{2}+\left(Y-\frac{1}{2} A \cos \theta_{2}\right)_{2} .
\end{gathered}
$$

By substituting these values of $R$ and $\phi_{2}$ into eq 22 and 24, and after choosing values for $X$ and $Y$, the changes in $X_{5}$ and $P$, for any rotation of the prism, are obtained.

Equation 22 may be written in the form

$$
X_{5}=\alpha+\beta \sin (\theta+\delta),
$$

where $\alpha \equiv X-Y$, and $\beta$ and $\delta$ are related by the expressions $\beta \quad \cos \delta \equiv R \quad\left[\sin \left(\phi_{2}-\theta_{2}\right)+\cos \left(\phi_{2}-\theta_{2}\right)\right]$, and $\beta \sin \delta \equiv R\left[\sin \left(\phi_{2}-\theta_{2}\right)-\cos \left(\phi_{2}-\theta_{2}\right)\right]+W_{2}+\frac{1}{2} A$. By eliminating $\delta$ from these equations, an expression relating $\beta$ and $R$ is obtained. A study of this relationship will show that when $\beta=0$, the value of $R$ is imaginary, except for the particular case when $\phi_{2}=\theta_{2}-\frac{1}{4} \pi$. Also, for this particular value of $\phi_{2}, R=\frac{1}{\sqrt{2}}\left(W_{2}+\frac{1}{2} A\right)$. When these values for $\phi_{2}$ and $R\left(=R_{2}\right)$ are substituted into eq 25 and 26, the resultant values for $X$ and $Y$ are found to coincide with the coordinates of the point $\left(X_{c}, Y_{c}\right)$, found by Bloch [4] and Uhler [2], for which there is no variation in $X_{5}$ as the prism is 

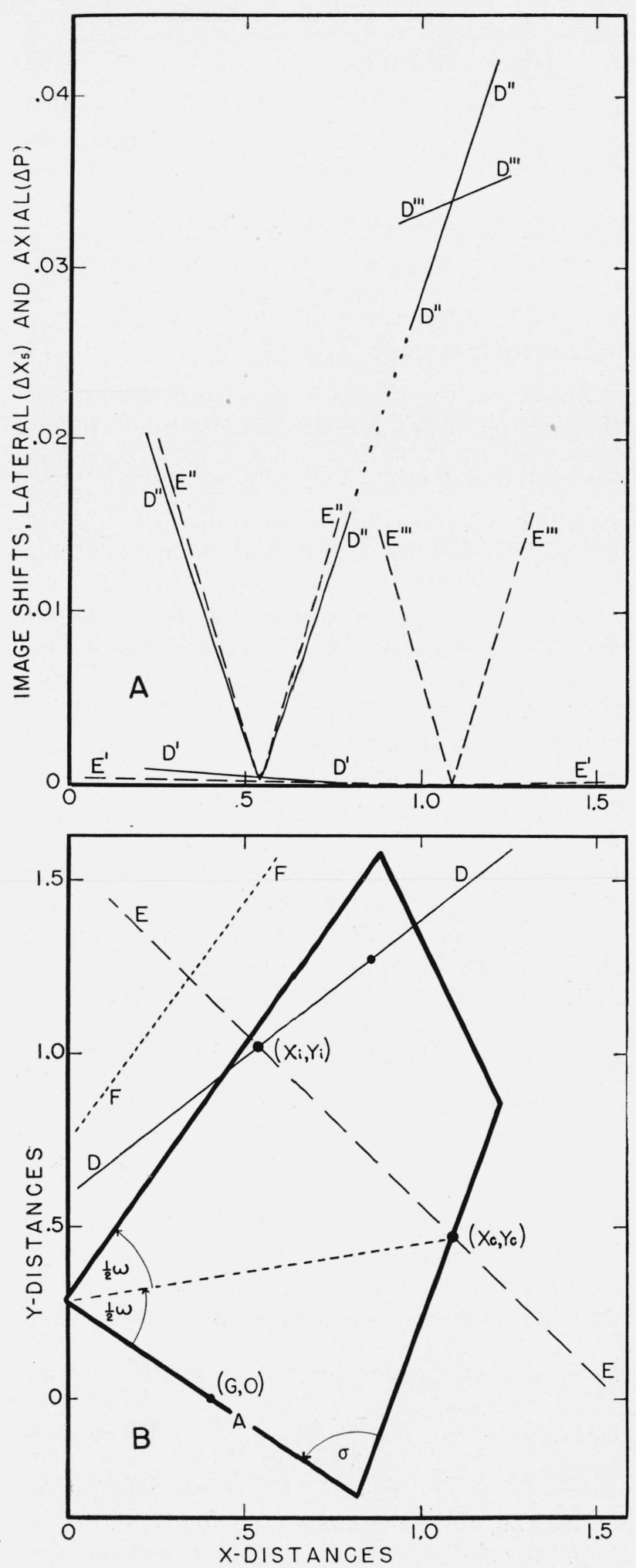

rotated. This point, designated here as the "critical point" (see fig. 3, B), is the intersection of the reflecting face of the prism with the bisector of the opposite angle, $\omega$, when the prism is in the position of initial conditions. For values of $\beta$ other than zero, the conditions for smallest change in $X_{5}$ require that a maximum or a minimum of $X_{5}$ must fall at $\theta=\theta_{2}$, which is midway between $\theta_{1}$ and $\theta_{3}$. This condition is realized by equating the value for $X_{51}$ to that for $X_{53}$, which yields the straight line, $X+Y=X_{c}+Y_{c}$, and which is represented by $E-E$ in figure $3, B$. If the axis of rotation is located at the critical point, then $X_{51}=X_{52}=X_{53}=$ constant for all values of $\theta$. The critical point, therefore, lies on line $E-E$.

While eq 24 does not exactly represent a simple sine curve, it does so to a close approximation over the limited range of rotations involved in this problem. Consequently, the above discussion relating to the variation in $X_{5}$, as shown by eq 22, applies equally well to the variation of $P$ as shown by eq 24 . The straight line $D-D$ in figure 3 bears the same relation to $P$ that line $E-E$ does to $X_{5}$. The point on $D-D$ that corresponds to the critical point is indicated by the dot above line $E-E$. The change in $P$ over the range $\theta_{1}$ to $\theta_{3}$ and for this point as axis of rotation is not zero but amounts to only a few microns if $A$ is less than $10 \mathrm{~cm}$. The intersection of $E-E$ and $D-D\left(X_{i}, Y_{i}\right)$ is designated as the "intersection point."

\section{Results}

The variation in $X_{5}$ with $\theta$, in the range from $54^{\circ}$ to $56^{\circ}$, is shown in figure $4, \mathrm{~A}$, for several points selected along the line $E-E$ as positions for the axis of rotation. Similarly, in figure 4, B, the same variation is shown for several points selected along the line $D-D$. The curves are labeled with the abscissas of the corresponding selected points. If the maxima and minima of a curve fall outside this range, as for curves labeled $X=0.525,0.531$, and 0.560 , the value of $X_{5}$

Figure 3. Relation of image shifts to position of axis.

A, Variation of image shifts, as the coordinates of the axis of rotation of the prism are changed; $\mathrm{B}$, initial position of the prism and the relative position of the axis of rotation for minimum image shifts. The abscissa scale is common to both parts of this figure. All scale values are based on the length of the entrance face as the unit. The loci of the axis of rotation for minimum image shifts with respect to the angle of rotation are: for the lateral shift, $E-E$, for the axial shift corresponding to the sagittal rays, $D-D$, and for the axial shift corresponding to the meridian rays, $F-F$. 

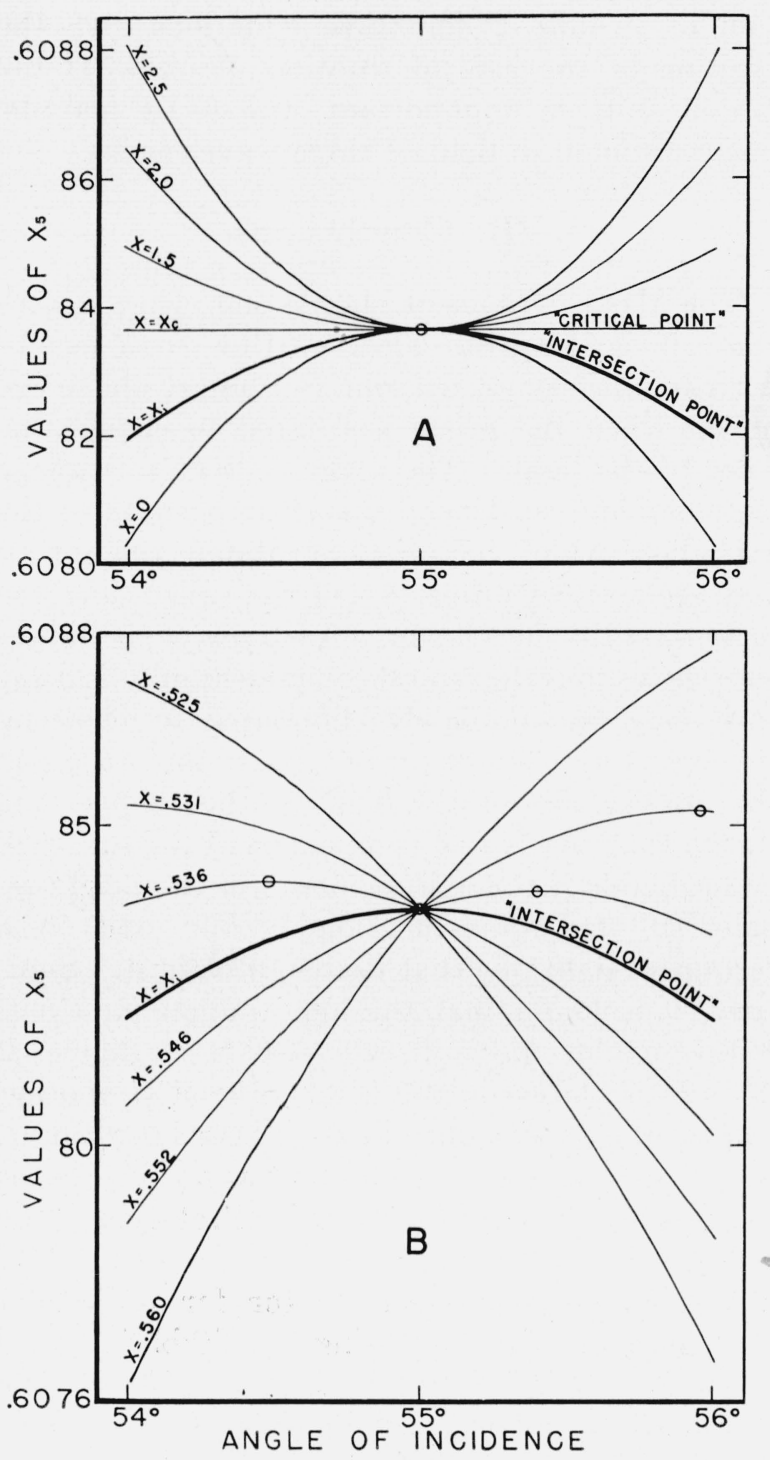

Figure 4. Variation in lateral position of image.

The change in the lateral position, $X_{5}$ of the image, with respect to $\theta$, as the axis of rotation is moved along line $E E$ of figure $3, \mathrm{~B}$, is given in figure 4, A. The same change in image position, $X_{5}$, as the axis of rotation is moved along line $D D$ of figure $3, \mathrm{~B}$, is shown in figure $4, \mathrm{~B}$.

increases or decreases throughout this range as the prism is rotated and the total absolute shift, $\Delta X_{5}$, equals $\left|X_{51}-X_{53}\right|$. If a maximum or a minimum (indicated by circles on some of the curves) falls within this range, say at $\theta=\theta_{m}$, the variation in $X_{5}$ is opposite for the two ranges, $\theta_{1}$ to $\theta_{m}$ and $\theta_{m}$ to $\theta_{3}$. If $\theta_{m}$ differs from $\theta_{2}$, the total absolute shift is the greater of the two values, $\left|X_{5 m}-X_{51}\right|$ and $\left|X_{5 m}-X_{53}\right|$. If $\theta_{m}=\theta_{2}$ (as for all curves in fig. $4, \mathrm{~A})$ the shift is $\Delta X_{5}=\left|X_{5 m}-X_{51}\right|=$
$\left|X_{5 m}-X_{53}\right|$, since, in this case, $X_{51}=X_{53}$ as was the requirement for the determination of line $E-E$.

The value of $\Delta X_{5}$ corresponding to all points along $E-E$ of figure $3, \mathrm{~B}$, is represented by the broken curve $E^{\prime}-E^{\prime}$ of figure $3, \mathrm{~A}$; the values for points along the perpendicular (not drawn) to $E-E$ at the intersection point are represented by $E^{\prime \prime}-E^{\prime \prime}$; and those for points along the perpendicular to $E-E$ at the critical point are represented by $E^{\prime \prime \prime}-E^{\prime \prime \prime}$.

The value of $\Delta P$ corresponding to all points along $D-D$ of figure $3, \mathrm{~B}$, is represented by the solid line $D^{\prime}-D^{\prime}$ of figure $3, A$; the values for points along $E-E$ are represented by $D^{\prime \prime}-D^{\prime \prime}$; and those for points along the perpendicular to $E-E$ at the critical point are represented by $D^{\prime \prime \prime}-D^{\prime \prime \prime}$.

All lines in figure $3, A$, are composed of one or more straight sections except $D^{\prime \prime}-D^{\prime \prime}$ and $E^{\prime \prime}-E^{\prime \prime}$. These have curvature only where their abscissas are in the neighborhood of the abscissa of the intersection point, $\left(X_{i}, Y_{i}\right)$. The nature of this curvature is shown more clearly in figure 5 by a sufficient enlargement of the scales. The corresponding curves are identified by their designations. The curvature is caused by the change in

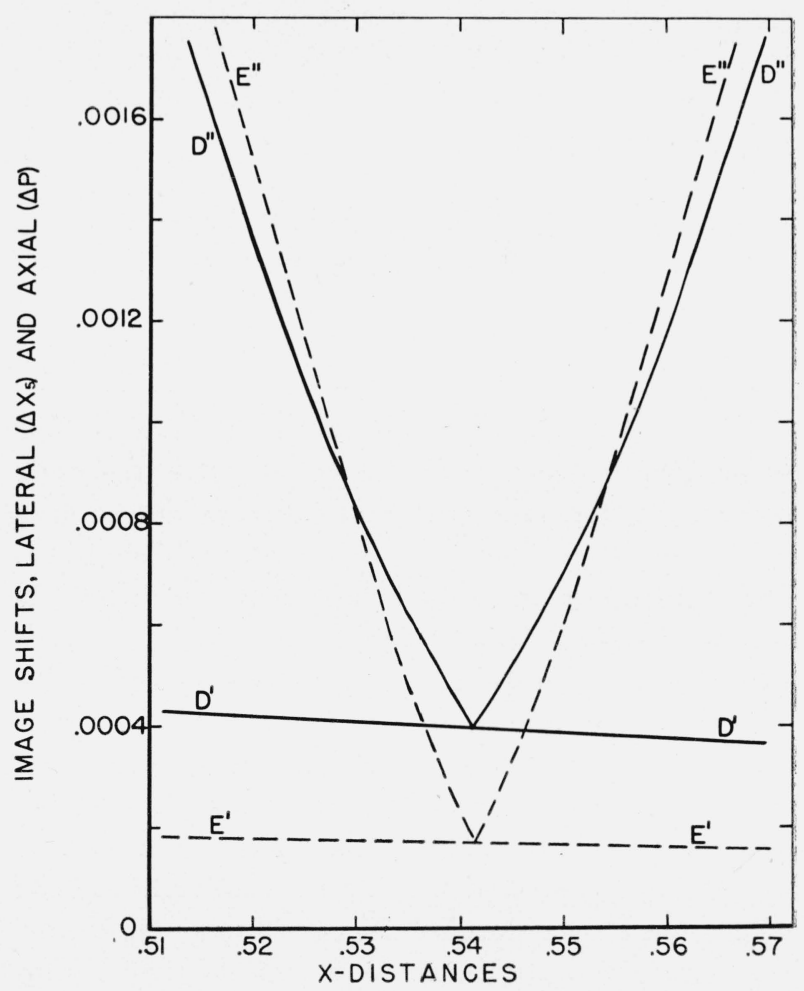

Figure 5. Enlargement of a small part of figure 3, A. 
the range covered by $\Delta X_{5}$ as $\theta_{m}$ moves through the range from $\theta_{1}$ to $\theta_{3}$.

As there is usually some error in placing the prism relative to the chosen axis of rotation, a study of figure $3, A$, will show that locating the axis of rotation at any point along line $E-E$ of figure $3, B$, and over the range shown by this figure should be about equally good insofar as $\Delta X_{5}$ is concerned. If the critical point should be chosen for the axis and an error of only $0.01 \times A$ were made in its placement, the resultant value for $\Delta X_{5}$ could be three times as large as it could be if the axis were accurately placed at the intersecting point. Also, an error in placement at the intersecting point that exceeds $0.02 \mathrm{~A}$ cannot produce a $\Delta X_{5}$ in excess of the largest $\Delta X_{5}$ that can be caused by an equal error of placement at the critical point. In the immediate neighborhood of the intersecting point the values for both $\Delta P$ and $\Delta X_{5}$ are small; values for $\Delta P$ (curve $D^{\prime \prime}-D^{\prime \prime}$ ) increasing rapidly as the axis moves along $E-E$ from this point and remaining almost constant (curve $\left.D^{\prime}-D^{\prime}\right)$ as the axis moves along $D-D$; values for $\Delta X_{5}$ (curve $E^{\prime \prime}-E^{\prime \prime}$ ) increasing rapidly as the axis moves from this point along $D-D$ and remaining almost constant (curve $E^{\prime}-E^{\prime}$ ) as the axis moves along $E-E$; and both increasing more or less rapidly for intermediate directions. Consequently, the point of intersection of $D-D$ and $E-E$ is chosen for the axis of rotation.

In case the meridian focus is dominant, the recommended point for the axis of rotation of the prism would be at the intersection of lines $E-E$ and $F-F$ (fig. $3, B$ ), since $F-F$ bears the same relation to the meridian rays that $D-D$ does to the sagittal rays.

This enables a designer to produce a monochromator consisting of a prism and a single achromatic lens for which the image distance is equal for three selected wavelengths of light. The treatment described here was used in the design of an instrument for the automatic recording of changes in interference phenomena [5].

A single simple lens may be used with a constant deviation prism to obtain a monochromator having a common focus for two selected wavelengths of light. By equating the right side of eq 22 , for $X_{51}$, to that for $X_{53}$ and the right side of eq 24 , for $P_{1}$, to that for $P_{3}$, two linear equations in $X$ and $Y$ are obtained. The simultaneous solu- tion of $X$ and $Y$ for these equations give the position of the axis of rotation desired. If the lateral shift is unimportant, the focus may be made common to light of three wavelengths.

\section{Conclusions}

The above treatment shows that when a constant deviation prism is used with a single lens to form an image of a fixed source or object, the image moves when the prism is rotated to change the color of the light. The magnitude of this image shift is found to depend upon the position of the axis about which the prism is rotated, the optical and geometrical properties of the prism, and the dimensions of the system. A survey of the literature shows that the lateral component of this image shift may be completely eliminated by choosing the axis at a certain point relative to the prism and the optical axis of the lens. In this paper it is shown that the lateral component of the shift will be negligible if the axis is chosen in the neighborhood of the above-mentioned point and on a certain straight line that passes through it. Similarly, it is found that the longitudinal (or axial) component of the shift will also be negligible if the axis is chosen in the neighborhood of another point and on a straight line that passes through it. These two straight lines intersect at a point that is conveniently located for the axis of rotation of the prism.

A fixed focus monochromator for ultraviolet work may be made by using a suitable isotropic crystal for the prism and collimator. This can be accomplished by placing the axis of rotation so that the change in effective object (or image) distance, produced by rotating the prism, is just sufficient to cancel the chromatic aberration of the lens.

\section{References}

[1] H. S. Uhler, Phys. Rev. 29, 37 (1909).

[2] H. S. Uhler, Astrophys. J. 47, 65 (1918).

[3] W. E. Forsythe, Astrophys. J. 45, 278 (1917).

[4] E. Bloch, J. phys. 7 [5], 145 (1917).

[5] J. B. Saunders, J. Research NBS 35, 157 (1945) RP1668.

[6] Southall's principles and methods of geometrical optics, p. 92 and 93 (The Macmillan Co., New York, N. Y., 1910).

[7] P. Pellin and A. Broca, J. phys. 8 [3], 314 (1899).

Washington, February 13, 1948. 